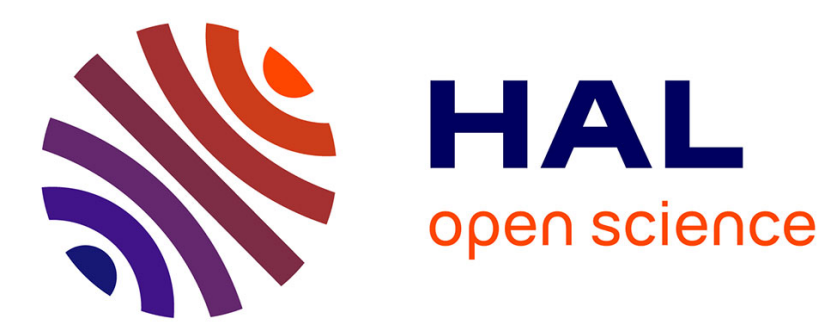

\title{
Le parti de Lincoln est-il devenu celui de Calhoun?
}

François Vergniolle de Chantal

\section{To cite this version:}

François Vergniolle de Chantal. Le parti de Lincoln est-il devenu celui de Calhoun?. Revue Française d'Etudes Américaines, 2002, 93 (3), pp.78. 10.3917/rfea.093.0078 . halshs-02508703

\section{HAL Id: halshs-02508703 \\ https://shs.hal.science/halshs-02508703}

Submitted on 15 Mar 2020

HAL is a multi-disciplinary open access archive for the deposit and dissemination of scientific research documents, whether they are published or not. The documents may come from teaching and research institutions in France or abroad, or from public or private research centers.
L'archive ouverte pluridisciplinaire HAL, est destinée au dépôt et à la diffusion de documents scientifiques de niveau recherche, publiés ou non, émanant des établissements d'enseignement et de recherche français ou étrangers, des laboratoires publics ou privés. 
François Vergniolle de Chantal

Article RFEA.

juin 2002.

Le parti de Lincoln est-il devenu celui de Calhoun?

Héritage sudiste, Parti républicain, et fédéralisme.

Aux Etats-Unis, un des événements politiques les plus marquants de ces dernières décennies est la radicalisation conservatrice du Parti républicain ${ }^{1}$. Si les observateurs se retrouvent pour la plupart sur ce constat, ils passent souvent sous silence un élément majeur de ce changement: une certaine vision commune de la constitution, et, en particulier, des rapports entre unités fédérées et Etat fédéral. Cette vision dépend en partie du conservatisme de la période antebellum, et, plus précisément, de la pensée de John Caldwell Calhoun (1782-1850).

La filiation heurte. Il est a priori délicat d'établir un lien idéologique entre le parti de Lincoln et les théories sécessionnistes: d'un côté le discours de la "maison divisée contre ellemême", de l'autre, la défense d'une vision confédérale. Et pourtant, l'objectif de cet article est de faire l'hypothèse selon laquelle c'est vers l'héritage sudiste, confédéré, qu'il faut se tourner pour saisir la réalité du changement opéré chez les républicains. Instrumentalisées par les sécessionnistes pour légitimer leurs objectifs politiques, les théories politiques de Calhoun sont, depuis lors, couramment perçues comme la conceptualisation la plus achevée de l'interprétation confédérée. Or actuellement, certains analystes n'hésitent pas à faire référence aux conceptions sudistes antérieures à la Guerre de Sécession pour expliquer les prises de position conservatrices dans le domaine du droit constitutionnel ${ }^{2}$. Comment expliquer cette mutation idéologique, alors que depuis la Guerre

\footnotetext{
${ }^{1}$ Pour un panorama général de la pensée conservatrice, cf George H. Nash, The Conservative Intellectual Movement in America since 1945, Wilmington, Delaware, Intercollegiate Studies Institute, 2nd éd. 1996 (1976), et, en français, Mokhtar Ben Barkha, La nouvelle droite américaine. Des origines à l'affaire Lewinski, Paris, Editions du Temps, 2nd éd. 1999 (1996).

2 Michael Lind, Up from Conservatism. Why the Right is Wrong for America, New York, The Free Press, 1996. Le chapitre 9, en particulier, s'intitule "The Confederate Theory of the Constitution" p.208-279. "The constitutional theory
} 
de Sécession, le Parti républicain a été le parti de la centralisation et les démocrates ont conservé en leur sein une branche sudiste éminemment conservatrice?

C'est essentiellement dans un calcul électoral délibéré du Parti républicain que réside la réponse. Et c'est dans le prolongement de cette tactique que les conservateurs ont habilement réévalué des théories constitutionnelles qui n'avaient plus cours depuis des décennies. Les récents arrêts de la Cour Rehnquist sur les relations intergouvernementales l'indiquent assez clairement; et le constat est encore plus frappant si l'on se penche sur les opinions dissidentes (dissent) des Juges les plus conservateurs, comme, par exemple, Clarence Thomas dans l'arrêt Thornton (1995). Dans cet article nous nous proposons de considérer le point de vue constitutionnel actuel des conservateurs en le liant aux théories confédérées antebellum. Cette hypothèse de travail est une occasion de retrouver la pensée juridique et philosophique d'un des plus brillants hommes politiques et penseurs de sa génération, John Calhoun. Décrié pour ses positions sur l'esclavage et sur la Sécession, discrédité pour son conservatisme intransigeant, il apparaît néanmoins comme une des références intellectuelles majeures de la droite. Sans être explicitement mentionné, il n'en est pas moins omniprésent.

La reconstitution de l'arrière-plan historique et intellectuel des prises de position constitutionnelles de la droite conservatrice va s'effectuer en trois temps. A partir de la stratégie électorale des dirigeants du Parti républicain, on pourra définir la notion de "fédéralisme dual" (dual federalism) et approfondir le référentiel juridique des juges conservateurs de la Cour. On retracera ensuite la genèse conceptuelle de cette théorie, dans le cadre de la pensée de Calhoun.

of the Republican right is not Republican in its origins; it is inherited, like so much else in today's Republican party, from the conservative Democrats of the South. (...) The Republican right, then, does have a venerable and consistent theory of the constitution. It just happens to be the Confederate theory" La citation est p.209. 


\section{La tactique électorale sudiste du Parti républicain: conséquences idéologiques d'un renversement des alliances.}

A partir du début des années soixante, les forces conservatrices montantes au sein des républicains orientent la tactique électorale du parti vers une reconquête des anciennes régions confédérées qui, à l'époque, sont de solides bastions démocrates. Pour comprendre les motivations qui poussent les dirigeants républicains, il faut remonter aux années trente. Le New Deal du président Roosevelt marque une grande rupture dans les perceptions partisanes ${ }^{3}$. Déjà réelle au cours des années vingt, l'orientation conservatrice du Parti républicain s'accroît encore. C'est tout particulièrement le cas en ce qui concerne l'interprétation du fédéralisme: les démocrates s'approprient explicitement le thème de la centralisation, alors que les républicains vont graduellement adopter une position favorable aux Etats fédérés.

Cette évolution républicaine sera clairement affirmée et revendiquée au début des années soixante. C'est en effet avec la stratégie sudiste de conquête de l'électorat blanc, évangélique et conservateur menée par le GOP (Grand Old Party) qu'apparaissent les premiers éléments de "l'antigouvernementalisme" républicain. Avec cette tactique, menée vigoureusement lors de la campagne présidentielle de 1964 sous la direction de Barry Goldwater, le parti tente pour la première fois de sortir de la marginalité dans laquelle l'omnipotence démocrate le cantonnait depuis des décennies. Après des débuts incertains, la manoeuvre sudiste s'avère être, à moyen terme, une réussite totale. L'électorat-cible, déstabilisé par la position anti-ségrégationniste du Parti démocrate, est largement réceptif aux avances du Parti républicain. L'inversion idéologique est alors complète ${ }^{4}$. Les républicains récupèrent les thèmes conservateurs propres aux blancs du Sud, notamment celui de la méfiance vis-à-vis du gouvernement fédéral, et une certaine défense des Droit des Etats contre les empiétements fédéraux 5 .

\footnotetext{
${ }^{3}$ A partir du New Deal, les démocrates donnent le ton du débat politique pour les trente ans à venir. En particulier sur la légitimité de la centralisation du pouvoir fédéral. Les républicains, encore largement liés à leur fondation liée au combat contre la Sécession, se placent dans une position de suivisme (les commentateurs évoquent ironiquement le "meetooism" du GOP, en particulier sous Eisenhower).

${ }^{4}$ Sur ce point, les sources sont nombreuses. Cf en particulier John G. Gerring, Party Ideology in America, 1828-1996, Cambridge, Mass., Harvard University Press 1998, Nicol C. Rae, Southern Democrats, Oxford, Oxford University Press, 1994, et, en français, Denis Lacorne, "La politique du soupçon d'immoralité: comparaisons franco-américaines", Pouvoirs, n63, mars 1993, p.89-98.

${ }^{5}$ Le livre maintenant classique de Kevin Phillips, jeune consultant politique républicain à l'époque, a énoncé cette tactique pour la première fois. Voir The Emerging Republican Majority, Arlington House, New Rochelle, New York,
} 
Le Parti républicain essaie d'attirer en son sein un électorat suburbain et conservateur en mettant en avant deux types d'arguments. D'une part des thèmes socio-religieux, destinés à satisfaire les attentes traditionalistes de cet électorat: la généralisation de la prière à l'école, l'interdiction absolue de l'avortement, la dénonciation des excès du féminisme, de l'homosexualité, de la pornographie et, plus généralement, la défense des valeurs familiales les plus traditionnelles. Second groupe d'arguments qui, pour être moins strictement moralisant, n'en sont pas moins très conservateurs: le refus de tout programme de discrimination positive (affirmative action, quotas), la remise en cause des programmes d'aide sociale, la libéralisation économique, et la réintroduction de la peine de mort. Les résultats ont été particulièrement probants, à tout le moins électoralement ${ }^{6}$. Alors que les républicains ne comptaient, en 1960, que sept Représentants (et aucun Sénateur) des Etats du Sud au Congrès, leur plus grande réussite électorale des dix dernières années a été menée par un républicain du Sud, Newt Gingrich. Une fois élu Président de la Chambre des Représentants (Speaker of the House), il lance toute une panoplie de réformes conservatrices qui font du 104ème Congrès (1994-96) un des sommets du retour conservateur depuis trente ans ${ }^{7}$.

C'est sur un mode d'expression, d'action et de problématisation axé autour de la critique de l'Etat fédéral que l'orientation conservatrice du Parti républicain trouve sa cohérence. En effet, ce thème est au coeur d'une conjonction d'intérêts entre les différentes mouvances du conservatisme. Toutes se retrouvent sur un thème global de critique contre le Big Government ${ }^{8}$. On peut déjà en donner une première approximation en se référant aux catégories de l'analyse classique menée par

\footnotetext{
3ème éd. 1970 (1969). Selon cet auteur, les populations des banlieues aisées, principalement localisées dans le Sud, sont un électorat porteur: foncièrement conservateurs, ces groupes ne peuvent voter pour un Parti démocrate en réorientation vers la gauche, et sont à la recherche d'un canal partisan différent.

${ }^{6}$ On peut remarquer dés maintenant que la coalition républicaine qui se met en place ressemble étonnamment à la tactique politique envisagée par Calhoun pour préserver, en son temps, l'équilibre social du pays. Pour lui, les conservateurs du Nord (surtout issus des milieux financiers et industriels) et du Sud (essentiellement de grands propriétaires terriens) devaient unir leurs forces contre les pressions progressistes issues du prolétariat urbain nordiste et du danger d'émancipation des esclaves sudistes. Ce parallèle, intéressant, ne concerne cependant pas directement le présent article. Sa formulation classique est faite par Richard Hofstadter dans le chapitre qu'il consacre à Calhoun, "The Marx of the Master Class", p.68-92, in The American Political Tradition, New York, Vintage Books, 1948. Pour une formulation contemporaine, cf Peter Applebome, Dixie Rising. How the South is Shaping American Values, Politics and Culture, Random House, Times Books, 1996, en particulier le chapitre 1, "Southernization of America", p.3-23.

${ }^{7}$ Sur ces réformes et leur impact immédiat, cf Alan Brinkley, Kathleen Sullivan et Nelson Polsby, The New Federalist Papers. Essays in Defense of the Constitution, New York, W.W. Norton, 1997. Sur l'humeur antifédérale actuelle et ses racines historiques, voir François Vergniolle de Chantal, "Le sentiment antigouvernemental aux Etats-Unis: essai de généalogie", Esprit, n²69, novembre 2000, p.119-135.

${ }^{8}$ Sur ce thème et son apparition au sein du Parti républicain, voir John Gerring, Party Ideology in America, op. cit., en particulier p. 135-142. Pour cet auteur, la dimension antigouvernementale du GOP est décelable dés les années vingt. De ce point de vue, la campagne de 1964 accélère une évolution entamée depuis un certain temps.
} 
George Nash. Pour les libéraux économiques, qui se retrouvent dans certaines franges de la nouvelle population suburbaine du Sud, pas de surprises, l'intervention de l'Etat est un mal, aussi bien au niveau fédéral que fédéré. Ils sont clairement les plus systématiques dans leur volonté de réduire les pouvoirs de l'Etat. Dans le cas du conservatisme plus traditionnel, l'attitude vis-à-vis de l'Etat central est ambiguë. En effet, le gouvernement fédéral est le seul acteur à avoir la possibilité de reprendre en main une société jugée corrompue. Lui seul pourrait imposer des valeurs substantielles pour pallier ce que le traditionalisme dénonce comme le vide de la société moderne. Mais en même temps, l'appareil gouvernemental est aux mains d'une élite libérale (au sens américain de centre gauche), laïque, méfiante à l'encontre de tout traditionalisme. Et c'est la raison pour laquelle la majorité des conservateurs traditionalistes préfère surtout réduire l'influence de cette élite nationale en confiant plus de responsabilités aux Etats fédérés 9 .

Ainsi, la conjoncture politique contemporaine façonne le conservatisme de telle sorte que son discours évoque les arguments de la période antebellum sur la question du Droit des Etats. C'est d'abord la convergence d'intérêts au sein de la droite contemporaine qui assure cette proximité. La reprise explicite de la tradition constitutionnelle sudiste n'apparaît que dans un second temps, en particulier lorsque la Cour Suprême, par le biais des Juges conservateurs, fait de la définition du fédéralisme une de ses préoccupations les plus fortes ${ }^{10}$. Par ailleurs, l'importance de l'héritage sudiste aide à comprendre la résonance historique des thèmes antigouvernementaux, autrement dit pourquoi ce type de discours joue à l'avantage des conservateurs dans le cadre de leur stratégie électorale depuis les années soixante. Il apparaît comme particulièrement porteur dans une région où, depuis la Guerre de Sécession, le gouvernement fédéral a été tenu dans une profonde suspicion. En fin de compte, la "sudisation" du Parti républicain est sensible à deux niveaux. D'abord comme la résultante d'une démarche consciente des dirigeants conservateurs du parti. Puis, dans un second temps, comme volonté de légitimation auprès de l'électorat blanc du Sud. De toutes les

\footnotetext{
${ }^{9}$ Chez Nash, les autres composantes du conservatisme (anticommunisme et droite religieuse des années soixante-dix par exemple), sont, elles aussi, fortement marquées par cette méfiance contre le gouvernement central. L'étude de leurs positions sort cependant du cadre de cet article.

${ }^{10}$ Différents arrêts récents indiquent une certaine recherche d'un "fédéralisme dual": National League of Cities v. Usery (1976), Gregory v. Ashcroft (1991), New York v. United States (1992), United States v. Lopez (1995), US Term Limits v. Thornton (1995), Seminole Tribe v. Florida (1996), Printz v. United States (1997), Alden v. Maine (1999), United States v. Morrison et al. (2000). Le "transfert" d'un thème politique dans la sphère juridique confirme encore une fois le diagnostic de Tocqueville selon lequel "Il n'est presque pas de question politique, aux Etats-Unis, qui ne se résolve tôt ou tard en question judiciaire", in De la démocratie en Amérique, 1981, Paris, Garnier-Flammarion, tome 1, p.370.
} 
manifestations de cette évolution, celle qui nous intéresse est la conceptualisation de type confédérée que le GOP met en avant.

\section{La recherche d'une théorie duale de la constitution.}

Qu'en est-il exactement de cette théorie? Quel a été son contexte de formation, quels sont ses principaux représentants et ses idées majeures? La littérature sur ce thème abonde. Paradoxalement, ce sont des historiens qui, en tentant de reconstruire le contexte des décennies précédant la Guerre de Sécession, ont donné sa formulation la plus concise à la vision confédérale classique $^{11}$.

Selon l'historien David Potter, la théorie dite "contractuelle" de la constitution, soutenant que l'Union était une ligue dans laquelle chacun des Etats signataires avait conservé sa pleine et entière souveraineté, est une dimension essentielle du conflit Nord-Sud. D. Potter part du constat que les Pères Fondateurs ont été particulièrement prudents dans leur projet constitutionnel en ce qui concerne les spécificités des Etats fédérés face au gouvernement central. Bien que nationalistes, ils ont délibérément laissé aux Etats une place de prédilection pour ménager les défenseurs de la souveraineté étatique. Leur prudence, nécessaire au vu de la difficulté du processus de ratification, a également donné des bases juridico-politiques aux arguments pro-étatiques antérieurs à la Guerre de Sécession. Il y a cinq points importants à retenir. D'abord la réalité politique et historique que constitue l'indépendance des colonies par rapport à la Couronne britannique. Le Traité de Paris (1783) reconnaît chacune des ex-colonies comme un Etat souverain et indépendant ${ }^{12}$. Et, de 1781 à la ratification de la nouvelle constitution, les Etats se sont regroupés en une Confédération dont la faiblesse essentielle, on le sait, a été de laisser trop de pouvoir aux Etats membres. Deuxième élément à prendre en considération, le mode de ratification du projet constitutionnel de 1787: il

\footnotetext{
${ }^{11}$ L'oeuvre principale en la matière est celle de David M. Potter, The Impending Crisis, 1848-1861, New York, Harper \& Row, Publishers, 1976. D'autres livres sont d'utiles compléments. En français, on peut renvoyer à la synthèse de Jean Heffer, L'Union en péril: la démocratie américaine et l'esclavage (1829-1865) Nancy, Presses Universitaires de Nancy, 1987. Plus théorique, et offrant des hypothèses intéressantes sur l'idéologie "States' Rights", est le livre de Richard E. Ellis, The Union at Risk. Jacksonian Democracy, States' Rights and the Nullification Crisis, New York, Oxford University Press, 1987.

${ }^{12}$ Une lecture nationaliste de ce traité reste cependant tout à fait possible. Dans la déclaration préliminaire, il n'est fait référence qu'aux "United States", et on évoque les Etats-Unis et la Grande-Bretagne en écrivant "the two countries" et non les...14. La liste des Etats peut être lue comme précisant le contenu géographique des "United States" libérés.
} 
devait être ratifié par 9 Etats pour être valable, ce qui est, bien sûr, une reconnaissance du rôle politique des Etats fédérés et, au-delà, une acceptation de la pluralité de la nation américaine, perçue, non pas comme un tout, unifié, mais d'abord comme une composition d'entités différentes 13. Au sein de la convention de Philadelphie elle-même, les options les plus nationalistes du projet de Virginie furent rejetées dés le départ. Ainsi, l'idée selon laquelle le gouvernement fédéral pourrait forcer un gouvernement étatique à agir de telle ou telle façon, a été explicitement abordée et refusée. A l'issue de la ratification, trois Etats (et non des moindres: outre le petit Rhode Island, la Virginie et New York sont dans ce cas), bien qu'ayant accepté le nouveau projet, ont introduit des clauses leur réservant le droit de reprendre, le cas échéant, les pouvoirs qu'ils venaient de déléguer ${ }^{14}$. Dernier élément, sans doute le plus important, la constitution repose largement sur les Etats fédérés en tant que tels, auxquels le texte accorde une place tout à fait éminente au sein des institutions. Le Sénat porte leur influence au coeur du processus décisionnel de l'Union, tandis que le Président est élu par un collège électoral basé sur la reconnaissance des Etats. Toute modification de la constitution doit avoir l'approbation des 3/4 des Etats. Plus tard, le Bill of Rights (décembre 1791), par son 10ème amendement, est une garantie supplémentaire aux Etats fédérés, puisqu'il reconnaît que "Les pouvoirs qui ne sont pas délégués aux Etats-Unis par la constitution ou refusés par elle aux Etats, sont conservés par les Etats ou par le peuple".

L'argumentaire confédéral repose sur la "théorie contractuelle" (compact theory) ou, autrement dit, sur une conception du "fédéralisme dual" (dual federalism) ${ }^{15}$. Pour J.F. Zimmerman,

13 De ce point de vue, le terme "We the People" prend un sens différent de celui de son interprétation nationaliste,
popularisée par Daniel Webster. Il fait référence non pas à un peuple uni, mais à treize peuples dans chacun des treize
Etats fédérés. En témoigne, outre la grammaire (We The People est utilisé au pluriel et pas au singulier), la première
rédaction du texte qui prévoyait d'énumérer chacun des Etats, du Nord au Sud. Ce choix fut abandonné après avoir
remarqué que l'on ne pouvait pas citer un Etat qui, in fine, rejetterait le projet. Cette première mouture, issue du Comité
de détail le 6 août 1787, est disponible in J.P. Kaminski, G.J. Saladino, The Documentary History of the Ratification of
the Constitution, vol.1, p.260-261. Par ailleurs, Madison, dans Le Fédéraliste n'39 corrobore largement cette thèse
selon laquelle ce sont les peuples des Etats, et non pas un peuple américain un et indivisible, qui détiennent le pouvoir
constituant. Voir aussi Lance Banning, The Sacred Fire of Liberty. James Madison and the Founding of the Federal
Republic, Ithaca, Cornell University Press, 1995 . En français, voir Thierry Chopin, La question de la souveraineté dans
la controverse constitutionnelle entre fédéralistes et anti-fédéralistes à la fin du XVIIIème siècle aux Etats-Unis: $1787-$
$88 "$, thèse de doctorat d'Etudes Politiques, Ecole des Hautes Etudes en Sciences Sociales, décembre 1999, qui offre une
perspective plus théorique sur cette question.
14 Dans le document de ratification adopté par la Virginie le 27 juin 1787 , on peut lire: "the powers granted under the
Constitution being derived from the people of the United States may be resumed by them whensoever the same shall be
perverted to their injury or oppression". Dans le cas de New York, le 26 juillet, il est précisé que "We the Delegates
declare and make known that the Powers of Government may be resumed by the People, whensoever it shall become
necessary to their happiness". Rhode Island, le 29 mai 1790 , a adopté la même clause que New York. Cité in David M.
Potter, The Impending Crisis, op. cit. p.482n77.
15 Il y a relativement peu de manuels qui soient entièrement consacrés à la question du fédéralisme, et en particulier sur 
le fédéralisme dual repose sur une série de quatre postulats juridiques, tels que le constitutionnaliste Edward S. Corwin a pu les présenter dans un article de 1950, et qui dessinent une image relativement statique du fédéralisme, organisé autour d'un strict partage des compétences entre les niveaux de gouvernement ${ }^{16}$. Les bases conceptuelles du fédéralisme dual sont les suivantes:

* Le gouvernement national a uniquement à sa disposition des pouvoirs définis, énumérés limitativement par la constitution (notamment dans l'article I section 8). Toute autre attribution est une déviation par rapport au texte constitutionnel.

* Dans la même perspective, les buts de ce gouvernement central ne peuvent être que fondamentalement limités, conformément à l'interprétation restrictive, défendue par Thomas Jefferson au début des années 1790, de la clause 18 section 8 de l'article 1, appelant à l'établissement des pouvoirs nécessaires et propres à l'exécution des compétences explicites du Congrès.

* La ligne de partage des compétences étant clairement établie, les deux niveaux de gouvernement -étatique et fédéral- sont souverains dans leur sphère respective. Autrement dit, ils sont, juridiquement, sur un pied d'égalité.

* La relation qui s'établit entre les deux gouvernements n'est pas harmonieuse. Elle est faite, au contraire, d'affrontements, de tensions, et rarement de collaboration.

Si la notion de fédéralisme dual créé certainement beaucoup plus de zones d'ombre qu'elle n'en supprime, il n'en reste pas moins qu'elle a réglé l'essentiel des rapports intergouvernementaux pendant soixante dix ans. Même si elle apparaît maintenant comme une interprétation juridique rigide des rapports fédéraux, elle prévalait néanmoins avant la Guerre de Sécession (avec les Cours Marshall, 1801/35, et Taney, 1836/1864), et ce sans être véritablement contestée. Les Etats fédérés étaient considérés comme le socle incontournable de l'Union et, comme tels, étaient des lieux

\footnotetext{
le cas américain. On peut renvoyer, en première approche, à Denis de Rougemont (eds), Dictionnaire International du Fédéralisme, Bruylant, Bruxelles, 1994, et à Maurice Croisat, Le fédéralisme dans les démocraties occidentales, 3ème éd., Paris, Montchrestien, 1999. Les manuels de science politique américain ont tous un ou plusieurs chapitres consacrés à cette question: cf James Q. Wilson, John J. Jr. DiIulio, American Government. Institutions and Policies, 7ème éd., New York, Houghton Mifflin Company, 1998, et Benjamin Ginsberg, Theodore Lowi, American Government. Freedom and Power, 6ème éd., W.W. Norton \& Company, 2000. Le livre le plus complet dressant un panorama de la question fédérale aux Etats-Unis, est Joseph F. Zimmerman, Contemporary American Federalism. The Growth of National Power, Praeger, Westport, CT, 1992. Les lignes qui suivent reprennent la définition donnée par l'auteur du fédéralisme dual (p.8). La revue éditée par le Center for the Study of Contemporary Federalism à Temple University, Publius, contient fréquemment des références intéressantes.

${ }^{16}$ L'article est republié in Richard Loss, Corwin on the Constitution. On Liberty against Government, vol. 3, Ithaca, Cornell University Press, 1988. Il est d'abord paru dans la Virginia Law Review, vol.36, n¹, p.1-23, février 1950.
} 
fondamentaux de décision politique. Les confédérés ont utilisé cette conception en la radicalisant et en niant l'existence d'un véritable peuple américain "trans-étatique". Une telle perspective peut sembler complètement dépassée de nos jours.

Pourtant, et c'est là notre hypothèse, c'est bien dans ce sens que doivent être interprétées les évocations actuelles du dualisme fédéral ${ }^{17}$. Dans un contexte général où les Etats sont fréquemment assimilés à des coquilles vides, dépossédés de tout pouvoir d'envergure par un Etat fédéral omnicompétent, on assiste bien au retour d'une interprétation de type "duale". L'exemple le plus fort de ce retour est sa discussion au sein de la plus haute instance juridique du pays, la Cour Suprême. Dans l'arrêt US Term Limits $v$. Thornton (1995) ${ }^{18}$, le dissent, rédigé par Clarence Thomas et auquel le Chief Justice Rehnquist, Sandra Day O'Connor et Antonin Scalia se sont joints, est d'un intérêt tout particulier en ce qu'il énonce clairement une conception confédérale des Etats-Unis. Pour eux, l'Arkansas a le droit de modifier les conditions d'élection de ses représentants au Congrès. Au nom de la souveraineté du peuple d'Arkansas, il est dit explicitement: "The ultimate source of the Constitution's authority is the consent of the people of each individual State, not the consent of the undifferentiated people of the nation as a whole". Autrement dit, il n'y a pas de peuple américain unique, indifférencié. Il y a 50 Etats qui retiennent une part de leur souveraineté. Ainsi, le fait que la constitution fédérale fixe les conditions d'éligibilité des membres du Congrès ne limite pas l'action des Etats. Elle fixe un plancher (âge, durée de résidence etc.) mais les Etats sont libres, au nom de leur souveraineté, de fixer d'autres conditions s'ils le désirent. L'apparition de ce type d'argumentation, même au niveau d'un dissent, est éminemment révélatrice du profond impact des thèses conservatrices et, surtout, du degré élevé de conceptualisation et de légitimité qu'elles ont atteint.

C'est pourquoi un bref retour s'impose sur le contexte de naissance de l'interprétation

17 Par exemple avec l'article de Jeffrey Rosen, "Dual Sovereigns: The Rehnquist Court Repudiates Congress, Reconsiders the New Deal and Resurrects States' Rights. So much for Judicial Restraint" in The New Republic, $\mathrm{n}^{\circ} 4306$, 28 juillet 1997, p.16-19. En français, on peut renvoyer à Sylvie Kauffmann, "L'Amérique en rébellion contre Washington", in Esprit, n²19, mars 1996, p.8-18. Elle fournit une présentation de l'enjeu politique contemporain autour des relations intergouvernementales. Ces deux références ne sont que des échantillons d'une masse sans cesse croissante de publications, et tout particulièrement dans le domaine du droit. Les Law Reviews se penchent de plus en plus sur ce thème, étant donné que la Cour Suprême, depuis une dizaine d'années, fait du fédéralisme un de ses sujets de prédilection.

${ }^{18}$ US Term Limits, Inc. v. Thornton, 514 US 779, 1995, opinion majoritaire délivrée par le Juge Stevens. L'arrêt invalide une loi de l'Arkansas qui limite le nombre de mandats que les Représentants de l'Etat en question peuvent accomplir au Congrès. 
confédérale. Ceci est d'autant plus vrai que peu de travaux ont été consacrés à la question du Droit des Etats. Parmi ceux-ci, l'étude menée par Richard E. Ellis donne un éclairage intéressant à la question de l'enjeu fédéral aux Etats-Unis ${ }^{19}$. Son hypothèse consiste à nuancer le jugement courant sur les States' Rights. Pour le sens commun universitaire, cet enjeu a été perçu comme la caractéristique de mouvements archaïques et/ou conservateurs qui, dés le début du XIXème siècle, tentent d'imposer une lecture duale rigide du fédéralisme américain. Cette perception associe le dualisme fédéral à une hérésie institutionnelle qui ne tient pas compte de la souplesse des institutions créées à Philadelphie. Elle constitue donc un obstacle à la consolidation nationale du pays telle qu'elle se met en oeuvre dans les premières décennies du XIXème. Or, une nuance est à faire entre deux types de perspective. La première, directement dans le sillage de la pensée antifédérale et des théories de Thomas Jefferson, ne renie pas l'Union et son caractère perpétuel. Dans cette optique, la plus courante, celle à laquelle le président Jackson fait référence lors de la crise de la Nullification, les States' Rights sont un moyen de décentraliser le pouvoir afin qu'il reste proche des citoyens. Ils sont une condition essentielle de maintien de la responsabilité politique et, de ce point de vue, doivent garantir le caractère démocratique du régime. Ces idées, modérées, "progressistes" dans le contexte de leur époque, s'opposent tout à fait à la seconde école qui, selon Ellis, organise pourtant elle aussi sa pensée autour du Droit des Etats, mais dans une optique tout à fait différente. Pour les partisans de cette thèse (Calhoun le premier), les States' Rights ouvrent la voie à une possibilité de sécession de l'Union. Celle-ci n'est qu'un contrat entre des unités étatiques souveraines, et elle ne résulte pas de l'existence d'un peuple américain unique, trans-étatique. Conçus comme un moyen de protéger les droits d'une minorité contre les exigences de la majorité numérique, les States' Rights vont être irrémédiablement liés à la cause sécessionniste et esclavagiste. L'histoire politique américaine des années 1830-60 repose, constitutionnellement, sur des oppositions entre les nationalistes et deux mouvements, que l'on peut appeler, après R. Ellis, les "traditionalistes" (vision classique des States' Rights), et les "nullifiers" appelés à devenir les avocats de la Sécession qui étaient aussi violemment en désaccord entre eux. C'est cette dernière tradition qui est destinée à prendre l'ascendant dans le débat politique américain ${ }^{20}$. Son impact est

\footnotetext{
${ }^{19}$ Richard Ellis, The Union at Risk, op. cit.

20 Sur ce thème, voir François Vergniolle de Chantal, "L'antifédéralisme américain. Diversité et pérennité", Commentaire, $\mathrm{n}^{\circ}$ 91, automne 2000, p.543-548. Par ailleurs, il faut se garder de faire du Sud un bloc idéologique uniforme. La réalité politique de l'époque ne peut se résumer aussi facilement. Le Sud des années 1830-60 n'est
} 
lié au traumatisme de la Sécession et ses échos se font encore sentir aujourd'hui, à l'inverse de l'autre tradition, qui, liée à des idéaux de républicanisme classique, s'est étiolée à partir du milieu du XIXème siècle, et dont l'héritage est à l'état de trace dans le paysage politique actuel. La tradition sudiste, elle, connaît, au contraire, un renouveau certain depuis les années 1960, avec, du fait de la stratégie électorale de conquête menée par le Parti républicain, la réintégration du Sud dans la vie politique. C'est pourquoi, pour mieux cerner la vision constitutionnelle du conservatisme courant, il est nécessaire de revenir sur la pensée du grand théoricien de l'école sécessionniste, John C. Calhoun.

\section{Genèse de la théorie confédérale.}

Vice-Président des Etats-Unis sous Jackson, puis Sénateur de Caroline du Sud, Calhoun a donné à l'idée de dualisme fédéral une radicalité qu'elle n'avait encore jamais eue. La vigueur qu'il a inspirée à une doctrine jusque là relativement modérée, constitue le socle intellectuel qui a fourni sa légitimité à la Sécession .

On sait qu'il s'est rendu tristement célèbre en affirmant notamment que l'esclavage était un bien positif ${ }^{21}$. Derrière cette affirmation se cache toute une argumentation conservatrice qui, critiquant la modernisation industrielle, affichant son pessimisme vis-à-vis de la nature humaine et, rejetant toute idée de droits naturels, peut présenter l'esclavage comme le résultat logique de ses présupposés ${ }^{22}$. En bref, la société patriarcale du Sud serait supérieure, économiquement, moralement et politiquement au système industriel basé sur des travailleurs blancs, salariés, tel qu'il se pratique au Nord. L'esclavagisme agraire est une garantie de stabilité, dans la mesure où il ne

nullement monolithique. Les Whigs ont gardé une certaine audience dans la région, tandis que les démocrates sudistes étaient encore nombreux dans l'entourage du Président Jackson. Au fur et à mesure de la radicalisation de l'enjeu de l'esclavage, cependant, l'éventail politique semble s'être considérablement réduit dans la région. Sur la naissance d'une certaine unité nationale sudiste qui tendrait à primer sur les divergences politiques, cf John McCardell, The Idea of $a$ Southern Nation: Southern Nationalists and Southern Nationalism, 1830-60, New York, 1979. Pour complément, se reporter à J. Mills Thornton II, "The Ethics of Subsistence and the Origins of Southern Secession", Tennessee Historical Quaterly, XLVIII, été 1989, p.67-85.

${ }^{21} \mathrm{Cf}$ "Speech on the Reception of Abolition Petitions", 6 février 1837, p.467, cité in Ross M. Lence, Union and Liberty. The Political Philosophy of John C. Calhoun, Indianapolis, Liberty Classic Edition, 1992.

22 Pour une mise en contexte de Calhoun et de sa pensée, cf Merrill D. Peterson, The Great Triumvirate, Webster, Clay and Calhoun, New York, Oxford University Press, 1987. On peut également utiliser, car ce travail est original par bien des aspects, John Niven, John C. Calhoun and the Price of Union, Baton Rouge, Louisiana State University Press, 1988; il s'agit d'une bonne biographie qui utilise la vie privée de l'homme pour discréditer certains lieux communs sur le personnage. Une anthologie des textes de Calhoun est disponible avec R.M. Lence, Union and Liberty, ibidem. 
laisse pas de place à l'antagonisme capital/travail. En ce sens, il préserve la stabilité d'une société dévouée aux idéaux républicains et à l'égalité, alors que l'industrialisme naissant dans le Nord corrompt ces idéaux et trahit le républicanisme. Ce retournement conservateur des thèses bien connues de Jefferson (autour de l'idéalisation du thème républicain classique) est, sans doute, un des aspects les plus marquants de la pensée de Calhoun ${ }^{23}$. Comme on le voit, l'aspect économique est lié à une dimension morale, la préservation de la vertu civique nécessaire au républicanisme. Au contraire, l'exploitation du prolétariat affaiblit les individus au point de les rendre dépendants et incapables de s'assurer comme citoyens à part entière. Déracinés, ils peuvent aisément être la proie des pires vices et, rapidement, constituer une source d'instabilité pour le républicanisme. L'harmonie du corps social est rompue. L'idéal de la société des "yeomen" est détruit, et, avec lui, le maintien d'une république authentique. Plus largement, et bien que se réclamant de Jefferson pour légitimer son point de vue (notamment sur le droit des Etats) ${ }^{24}$, Calhoun réfute pourtant les idéaux des Lumières, par exemple en ce qui concerne la philosophie des droits naturels.

Ce cadre général, éminemment conservateur, axé sur la dénonciation de la société industrielle, se double d'un rejet absolu de toute tentative du pouvoir central d'empiéter sur les Etats. La consolidation de la république américaine n'est qu'une manifestation de plus de l'extension de la corruption sociale. Tout approfondissement du seul règne de la majorité numérique va dans le sens de la corruption. Le système des dépouilles officialisé et généralisé sous Jackson semble, pour Calhoun, en être la preuve la plus flagrante. La politique démocratique dégénère en luttes partisanes pour le contrôle des machines électorales ou les rétributions de toutes sortes que peut donner le gouvernement central. De plus, l'instauration d'un système partisan national réduit à néant ce qui est, selon Calhoun, un élément essentiel du mécanisme institutionnel américain, le principe de l'équilibre constitutionnel. En effet, le bipartisme paralyse toute résistance et toute autonomie

\footnotetext{
${ }^{23}$ Cf Robert E. Shalhope, "Thomas Jefferson's Republicanism and Antebellum Southern Thought", in The Journal of Southern History, vol. XLII, ${ }^{\circ} 4$, novembre 1976, p.529-556. Selon lui, ce qui primait pour Jefferson était la préservation des conditions sociales et économiques qui président au républicanisme. Or, l'évolution industrielle de la société au Nord va convaincre Jefferson de la menace de corruption qu'elle fait peser. De plus en plus, Jefferson ressent que c'est le Sud qui incarne véritablement les idéaux républicains et agraires. En fin de compte, face à la corruption de la société, il ne resterait qu'une seule barrière, institutionnelle et, plus précisément, le Droit des Etats. Cette évolution conservatrice du républicanisme jeffersonien constitue une puissante source de légitimité pour les penseurs sudistes ultérieurs.

${ }^{24}$ Pour une analyse complète de la volonté de Calhoun de se légitimer comme héritier de Jefferson, cf Merrill D. Peterson, The Jefferson Image in the American Mind, New York, Oxford University Press, 1960; voir en particulier les p.51-66.
} 
véritable du gouvernement des Etats fédérés. On peut ainsi déboucher sur une forme de tyrannie menée par une majorité animée par un seul intérêt partisan.

Calhoun part du constat selon lequel, dans toute société, il existe un combat incessant entre un parti majoritaire et un autre, minoritaire, autour du contrôle du gouvernement. Dans une démocratie, ce conflit s'arbitre selon la règle du choix numérique/populaire, régulée par les contraintes légales imposées par la constitution. Mais ceci est insuffisant pour s'opposer à la dérive centralisatrice et démocratique que dénonce Calhoun. Selon lui, en plus d'une reconnaissance de la majorité du nombre, il doit impérativement y avoir une reconnaissance de celle des intérêts géographiques (autrement dit, concrètement, des Etats fédérés). Ces derniers, doués du pouvoir d'arrêter les déviances de la majorité populaire, devraient, de ce fait, assurer la réalisation d'un bien commun où la majorité ne peut brimer la minorité.

On comprend alors que Calhoun se soit posé comme le défenseur acharné de la souveraineté étatique qui, à ses yeux, apparaît comme étant le rempart aux dérives majoritaires, centralisatrices et égalisatrices qui se font jour dans le Nord du pays. Il a donc porté le droit des Etats à son plus haut niveau de conceptualisation. Dans A Discourse on the Constitution and Government of the United States (1850), il donne une formulation parfaitement explicite de sa vision confédérale des EtatsUnis: "Ours is a system of governments, compounded of the separate governments of the several States composing the Union, and of one common government of all its members, called the Government of the United States. The former preceded the latter, which was created by their agency" 25 . Dans le cours de ce raisonnement, plusieurs élément intéressants sont abordés, en particulier la question de la Sécession et la possibilité de protéger les Etats per se en leur donnant plus de poids dans la structure fédérale. En ce qui concerne le premier point, l'attitude de Calhoun est relativement nuancée, et, même s'il est plus que réservé vis-à-vis d'une désunion, il n'en exclut pas la possibilité logique. En effet, sa conception des Etats-Unis est celle d'un contrat entre des Etats souverains. Dans cette optique, rien de plus normal qu'une rupture du contrat par une des parties prenantes (un des Etats fédérés), en cas de modification des termes par l'autorité centrale: "That a State, as a party to the constitutional compact, has the right to secede -acting in the same capacity in which it ratified the constitution -cannot, with any show of reason, be denied by any one

\footnotetext{
${ }^{25}$ R.M. Lence, Union and Liberty, op. cit. p.81.
} 
who regards the constitution as a compact- if a power should be inserted by the amending power, which would radically change the character of the constitution, or the nature of the system; or if the former should fail to fulfil the ends for which it was established" ${ }^{26}$. Les Etats, dans un système fédéral, en constituent la structure principale et la source première de légitimité. Or, selon Calhoun, les institutions existantes (Sénat essentiellement) sont inefficaces pour garantir un poids suffisant aux unités fédérées. C'est ainsi qu'il propose un aménagement institutionnel nouveau, celui d'un exécutif bicéphale, qui représenterait à la fois la majorité numérique, avec un président élu par les citoyens, et les intérêts territoriaux, avec un "président-bis", chargé de préserver les droits des Etats fédérés en imposant son veto à toute mesure trop centralisatrice décidée par la simple majorité numérique. Comme il le dit, "Its powers (ceux de l'exécutif), instead of being vested, as they now are, in a single officer, should be vested in two -to be so elected, as that the two should be constituted the special organs and representatives of the respective sections, in the executive department of the government, and requiring each to approve all the acts of Congress before they shall become laws" ${ }^{27}$. En fin de compte, Calhoun propose, de créer un nouvel organisme, inconnu des théories constitutionnelles de Jefferson et de Madison. Le sommet de l'Etat serait une véritable dyarchie avec un titulaire qui serait l'élu du peuple, et un autre qui serait celui des Etats. Chacun ayant le droit de veto nécessaire pour empêcher l'autre de faire une politique contraire aux intérêts de ses mandants. Ce mécanisme de "concurrent majority" (aussi appelée "constitutionnelle") constitue la grande innovation institutionnelle défendue par Calhoun. La concurrent majority se comprend en fait comme un outil de prévention de l'oppression de la minorité par la majorité populaire, ou, pour le dire autrement, le mode d'expression des Etats per se au sein d'une fédération démocratique. Elle a par ailleurs une vertu positive en ceci que, comme elle nécessite le consentement de chacun des intérêts géographiques pour mettre en oeuvre une politique gouvernementale, elle engendre forcément le consensus une fois que la décision est prise. Euphémisme pour désigner la nécessité d'un consentement unanime, cette théorie semble

\footnotetext{
${ }^{26}$ R.M. Lence, Union and Liberty, ibidem, p.212.

27 R.M. Lence, Union and Liberty, ibid, p.275. Pour des approfondissements sur la conceptualisation de la majorité concurrent, ef Lacy K. Ford, "Inventing the Concurrent Majority: Madison, Calhoun, and the Problem of Majoritarianism in American Political Thought", The Journal of Southern History, vol.60, n¹, février 1994, p.19-58. Du même auteur, "Recovering the Republic: Calhoun, South Carolina, and the Concurrent Majority", South Carolina Historical Magazine, vol.89, 1988, p.146-159. Voir aussi, Pauline Maier, "The Road Not Taken: Nullification, John C. Calhoun, and the Revolutionary Tradition in South Carolina", South Carolina Historical Magazine, vol.LXXXII, 1981, p.1-19.
} 
difficilement conciliable avec une capacité d'action efficace. L'exemple pris par Calhoun, celui des jurés lors d'un procès, où il est nécessaire que les douze personnes s'entendent pour un seul et même verdict, illustre parfaitement les impasses auxquelles peut mener sa proposition en soulignant le côté procédurier de la concurrent majority. Peut-on déduire du mode de fonctionnement juridique un principe d'action politique, alors que les exigences des deux domaines ne coïncident pas forcément? Ce problème, délibérément contourné dans les textes du Sénateur de Caroline du Sud, fait sentir l'irréalisme de sa position. Tenaillé par une exigence de justice et d'impartialité, il est conduit à passer sous silence les exigences d'efficacité que comporte la vie politique concrète.

D'autre part, l'Histoire fournit quantité d'exemples (de l'Antiquité romaine à la Renaissance italienne) où des collèges exécutifs ont été institués avec les meilleures intentions du monde (équité, contrôle, transparence etc.), pour n'aboutir qu'à la confusion, à l'inefficacité et, au final, à la fin du régime. On atteint là les limites de la pensée calhounienne qui, puissamment efficace dans son analyse des logiques du système fédéral, est incapable de fournir une alternative crédible aux évolutions qu'elle diagnostique. C'est d'ailleurs cette même impasse pratique, concrète qui est présente dans A Disquisition on Government. Elle est d'autant plus sensible que c'est dans ce texte que les principes de base de la théorie politique de J.C. Calhoun sont développés, en particulier l'idée de la concurrent majority ${ }^{28}$.

Pourtant, c'est encore dans ce sens de la dispersion du pouvoir que Calhoun développe l'essentiel de sa théorie. On pourrait dire que Calhoun a délibérément inséré, en l'accentuant, une thématique de type "poids et contrepoids" dans une problématique fédérale en attribuant aux Etats qui composent le Sénat, le statut que John Adams réservait à l'aristocratie ${ }^{29}$. Pour Calhoun, la garantie la plus efficace, pour tout gouvernement républicain, n'est pas seulement, comme chez Adams, un équilibre entre des ordres et des classes sociales. Ce point de vue, qui remonte aux théories de la constitution mixte chère à l'Antiquité, doit être complétée. Il faut y ajouter les

\footnotetext{
${ }^{28}$ Les deux textes constituaient les ébauches d'un grand traité général sur le gouvernement. Ce projet n'a jamais pu être mené à bien. Le Discourse (..) présente une analyse de la nature politique des Etats-Unis, définis comme une union confédérale dans laquelle un exécutif bicéphale devrait être institué. Quant à Disquisition (...), premier texte politique de Calhoun -mais, paradoxalement, publié après sa mort-, il s'agit d'un texte théorique où la doctrine de la concurrent majority est expliquée.

${ }^{29}$ Sur John Adams et la tradition de checks and balances aux Etats-Unis, cf Bernard Manin, "Frontières, freins et contrepoids. La séparation des pouvoirs dans le débat constitutionnel américain de 1787", Revue Française de Science Politique, vol.44, n², avril 1994, p.257-293.
} 
divisions géographiques. Ainsi un authentique système de checks and balances est garanti ${ }^{30}$.

L'Etat local est donc la base de toute la théorie de Calhoun, et ce parce qu'il y voit le meilleur rempart contre les tendances consolidatrices du pouvoir central, entendu que, pour lui, ce terme est synonyme de "corruptrices". Toute conception des Etats-Unis comme une Nation à part entière est une erreur fondamentale ${ }^{31}$. Les Etats-Unis sont en fait une "Nation of States" ${ }^{32}$, difficilement analysable selon des critères européens où le modèle de l'Etat-Nation unitaire règne en maître. Pour ses défenseurs, Calhoun puise aux sources de l'idée fédérale. Il se situe tout à fait dans le prolongement de la définition classique de Montesquieu écrivant: "Cette forme de gouvernement (la république fédérale) est une convention par laquelle plusieurs corps politiques consentent à devenir citoyens d'un Etat plus grand qu'ils veulent former. C'est une société de sociétés qui en font une nouvelle qui peut s'agrandir par de nouveaux associés qui se sont unis ${ }^{33}$. Or il s'agit, en fait, d'une définition de type confédéral (où les composantes restent souveraines), et non pas fédéral où une part de souveraineté est effectivement transférée au niveau supérieur. Dans ce cadre, il est clair que la ligne rouge de la sécession est aisée à franchir.

Actuellement, il va de soi que le Parti républicain a une interprétation constitutionnelle bien plus modérée. Le récent dénouement du feuilleton électoral de la présidentielle 2000 atteste de la fragilité de l'engagement républicain sur les questions de fédéralisme. Les Juges conservateurs n'ont pas hésité à frôler l'incohérence vis-à-vis de dix ans de jurisprudence sur le fédéralisme en annulant directement la décision de la Cour Suprême de Floride ordonnant le recompte général des voix. Sur

\footnotetext{
${ }^{30}$ Avant que ce thème ne connaisse son apogée avec Calhoun, il revient à un jeune conservateur de Virginie, du nom d'Abel P. Upshur, d'énoncer, dans un traité sur la souveraineté étatique publié en 1840, le principe fondamental qui va orienter toute la pensée de Calhoun sur ce point: "There is a majority of interests as well as a majority in number". On peut aussi voir une certaine anticipation dans la définition qu'Orestes Brownson donne de la démocratie américaine, la désignant comme une "démocratie territoriale" et non pas seulement une démocratie numérique, ou majoritaire. Sur cette figure paradoxale qu'est Orestes Browson, cf Naomi Wulf, L'idée de démocratie aux Etats-Unis, de 1828 à 1844, à travers les écrits d'Orestes A. Brownson", Thèse de doctorat d'Anglais, Lille III, 1996.

${ }^{31}$ Cf Samuel H. Beer, To Make a Nation: The Rediscovery of American Federalism, Cambridge, The Belknap Press of Harvard University Press, 1993, p.8 explique ainsi que: "In the course of the struggle with sectionalism, John C. Calhoun defined the issue and threw down the challenge to nationalism when he said: "the very idea of an American People, as constituting a single community, is a mere chimera. Such a community never for a single moment existedneither before nor since the Declaration of Independence." This was a logical deduction from the compact theory, which in Calhoun's system made of each state a "separate sovereign community"". Le livre est en grande partie une discussion des grandes théories qui ont présidé à l'élaboration du fédéralisme américain. S'y ajoutent les divergences entre les interprétations fédéralistes et républicaines. L'auteur cherche surtout à légitimer l'action et la pensée "fédéraliste".

${ }^{32}$ Nous faisons ici allusion au livre de Robert A. Goldwin, A Nation of States. Essays on the American Federal System, 2ème éd. 1964, Chicago, Rand Mc Nally \& Company. Il s'agit d'une série d'études sur les différents aspects du système américain, et les principales controverses qu'il a pu susciter.

${ }^{33}$ Montesquieu, Esprit des lois (tome I, p.137), cité in Maurice Croisat, op. cit. Souligné par moi.
} 
ce point, l'équipe de Bush Junior n'a pas cru bon de s'en prendre à l'interventionnisme fédéral! Néanmoins, même si les thèses calhouniennes, d'obédience confédérales, ne sont reprises que sous une forme atténuée, les résonances sudistes sont belles et bien présentes dans le discours républicain contemporain, notamment en ce qui concerne l'interprétation constitutionnelle. Depuis la nomination de Rehnquist comme Chief Justice en 1986, ce sont bien les Juges conservateurs de la Cour (nommés par Reagan et Bush) qui semblent mettre en avant un authentique projet de redéfinition des relations intergouvernementales dans le sens d'une lecture duale. Le 104ème Congrès (1994-96), a constamment affiché sa volonté de défendre les Etats fédérés contre le gouvernement central. En fin de compte, le conservatisme actuel utilise à son profit une authentique rhétorique confédérale qui, bien que souvent réduite à une pure et simple évocation, a eu, il y a peu, de clairs effets en termes de politiques publiques. La loi de réforme de l'assistance sociale (Personal Responsibility and Work Opportunity Reconciliation Act, 1996) est un transfert réel de compétence du gouvernement central aux Etats fédérés ${ }^{34}$. Dans le même temps, la jurisprudence de la Cour Suprême (en particulier par son interprétation restrictive de la clause commerciale et du XIVème amendement) oblige le gouvernement fédéral à redéfinir son mode d'action. En effet, de nombreuses régulations fédérales semblent maintenant, au vu de la nouvelle orientation jurisprudentielle, contestables devant les tribunaux. Ce revirement de la Cour Suprême, aux conséquences potentiellement importantes, trouve une de ses sources chez Calhoun, même si l'actualité récente incite à nuancer le poids réel de cet apport ${ }^{35}$.

Reste que la raison qui, de nos jours, pousse les conservateurs actuels à puiser à cette source demeure surprenante. Mise à part la question de la tactique électorale du Parti républicain contemporain et sa volonté de raffermir son audience dans la région, il semble difficile de concevoir l'existence d'un lien idéologique entre le parti qui a mené la Guerre de Sécession pour préserver

\footnotetext{
${ }^{34}$ Sur cette loi, voir Daniel Béland, "La fin du Welfare State", Esprit, n²32, mai 1997, p.38-58. Pour une mise en contexte plus générale, voir également Daniel Béland, François Vergniolle de Chantal, "Politiques sociales, stratégies électorales et fédéralisme sous la Présidence Clinton", Revue Française de Science Politique, vol.50, nº, décembre 2000, p.883-913.

${ }^{35}$ Cet article met volontairement l'accent sur la dimension calhounienne dans le Parti républicain. L'héritage antifédéraliste, autrement dit, celui des opposants au projet de Philadelphie en 1787-88, devrait aussi être pris en compte. Sur les rapports entre Calhoun et les anti-fédéralistes, voir V. de Chantal, "L'antifédéralisme américain", art. cit.
} 
l'Union, et les idées du plus grand penseur de la théorie confédérale.

En fait, le fossé intellectuel est tel qu'il permet de comprendre l'ampleur de la mutation du Parti républicain depuis les années soixante. Bien loin d'avoir toujours été le parti conservateur que l'on connaît maintenant, il a, au contraire, été bien souvent à la pointe de luttes progressistes (de l'émancipation, son acte fondateur, au Progressisme des années 1900). C'est dire la force du renouveau de la droite depuis trente ans. L'émergence de théories néo-calhouniennes au sein du Parti républicain est une des preuves les plus visibles du virage idéologique qui caractérise le paysage politique américain depuis les années soixante. Même si la décennie qui vient de s'écouler est marquée par un certain recentrage (comme en témoignent la recherche clintonienne d'une "Troisième Voie" ou le "conservatisme à visage humain" de Bush junior), l'impact de la radicalisation menée par les dirigeants conservateurs du Parti républicain est toujours sensible. Le parti de Lincoln comporte en son sein une large frange extrêmement conservatrice, géographiquement attachée au Sud, et plus que jamais désireuse d'imposer ses vues: les compromis que Bush junior a dû faire pendant les primaires de 2000 (en intervenant notamment à l'université ultra-conservatrice Bob Jones en Caroline du Sud), sont des preuves de la permanence du poids de la branche sudiste dans le parti.

Mots-Clés

Calhoun (Calhoun)

Confédération (Confederation)

Droit des Etats (States’ Rights)

Fédéralisme (Federalism)

Parti républicain (Republican Party)

\begin{abstract}
The criticism against Federal Government is one of the defining characteristics of the Republican Party. But analysts usually fall short of explaining it. This article attempts to analyze the
\end{abstract}


antigovernmental mood of the American Right by linking it to the confederate theories of antebellum America. More specifically, it tries to show that Calhoun's theories are present in some aspects of the current conservative constitutional analysis, especially in the writings of Justice Clarence Thomas. It then moves on to an account of Calhoun's constitutional views as well as his assertion that America lacked a true national identity before the Civil War. These ideas - still largely forgotten - are nonetheless crucial in understanding important parts of the conservative intellectual renewal.

\section{Abstract}

For any analyst of American politics, the critique against Federal Government is one of the main characteristics of the Republican Party.

**** i would say "criticism of the" instead of "the critique against" -- do you mean criticism or evaluation? if you just mean that they examine or evaluate/finetune the government then critique is fine. I also think that that characteristic holds true in just about anyone's mind, whether they're an analyst of American politics or not, so really the opening phrase is unnecessary. "defining" would be a stronger and maybe a better word than "main" -- if you use that, then you may be in specialist territory as opposed to knowledge generally possessed by the public. but, you may not agree that it is a "defining" characteristic.

But they usually fall short of accounting for it.

**** i'm not really sure what you mean by that -- you mean accepting blame for its shortcomings? this isn't an english question, but i'm not sure how this sentence relates to the rest of the abstract. maybe you mean "explaining it" instead of "accounting for it" -- if so you'll have to find a way to say that. also, it is clear to your average reader that by "they" you mean Republicans, although grammatically it is not exactly clear since there is no plural noun preceding this sentence except maybe "characteristics" -- "Party" is actually just a singular noun. i'm not sure how i might fix that.

This article tries to analyze the antigovernmental mood of the American Right by linking it to the confederate theories of antebellum America.

**** again, not really an english question, but do you need to say "tries"? can you just say "this article analyzes..."? anyway i prefer the word "attempts" rather than "tries." the "tries" in the next sentence is fine i would say, especially if 
you change the first "tries" to "attempts".

More

specifically, it tries to show that Calhoun's theories are

present in some aspects of the current conservative

constitutional analysis, especially in the writings of

Justice Clarence Thomas. It then moves on to an account of

Calhoun's constitutional views as well as his questioning

of a true American national identity before the Civil War.

**** this is fine, although "his questioning of" is (because of the gerund, etc.) maybe not as good as an alternate wording like "as well as his assertion that America lacked a true national identity before the Civil War." if you use this longer

wording then it might be helpful to say "It then moves on to an account of Calhoun's constitutional views and of his assertion...." that way the second phrase is more clearly delineated.

These ideas - still largely forgotten - are nonetheless

crucial in understanding important parts of the

conservative intellectual renewal. 